\title{
MODELING AND CONTROL OF SWITCHED RELUCTANCE MOTOR DRIVES BASED ON SETPOINT RELAY
}

\author{
Laurinda L.N. dos Reis*, A.A.R. Coelho**, O.M. Almeida* and R.N.C. Almeida* \\ * Federal University of Ceará - DEE/GPAR - www.dee.ufc.br - Fax:+55-85-33669574 \\ ** Federal University of Santa Catarina - UFSC-SC \\ laurinda@ufc.br, aarc@das.ufsc.br, otacilio@dee.ufc.br,rnunes@dee.ufc.br
}

\begin{abstract}
This paper considers a novel approach to the accurate on-line modeling of a switched reluctance motor (SRM) drive. Moreover, the implementation of a simple autotuning technique for SRM current control based on PWM controllers using modified Ziegler-Nichols tuning method is also introduced. The proposed method is an extension of the relay feedback control. It uses a setpoint relay framework to extract relevant on-line information about the process dynamics. The proposed PWM controller has been simulated and a hardware prototype was then implemented with digital signal processor control to evaluate the method using a 12/8, 3-phase SRM. The experimental results of the drive model validate the introduced current control method.
\end{abstract}

Keywords - Switched Reluctance Motor, Relay Modeling, Experimental Identification.

\section{INTRODUCTION}

Switched reluctance motors (SRMs) are potentially attractive for many industrial applications due to their simple structure, high speed, and low cost. However, as SRM utilization in industry is still relatively limited compared to other machine types, the practical drive implementation issues have not yet fully explored.

One of the major disadvantages of SRMs is the torque ripple. The torque is often controlled via an inner current loop, with a nonlinear relationship between torque and current. To achieve good torque linearity and low torque ripple, accurate command tracking is required from the current controller. At low speed, current profiling may be utilized to reduce torque ripple. Several researchers have proposed current profiling based methods to minimize torque ripple ([6], [4] and [2]). The SRMs have been traditionally controlled by either open-loop hysteresis or closed-loop pulse-width-modulation (PWM) current controllers. Each scheme has its advantages and drawbacks with regard to insensitivity to parameters variations, accuracy, robustness, and dynamic response over the entire speed range. The advantages of conventional hysteresis current controllers lie in their simplicity and capability of providing fast responses and good accuracy, because they act quickly. However, there are well known disadvantages such as large current ripple and a widely varying

Manuscript received November 5, 2007; revised March 7, 2008. Recommended by the editor F.L.M Antunes. switching frequency. Although there are some techniques to solve the frequency issue, these problems usually make the controller unfit for many applications. On the other hand, PWM controllers provide improved characteristics of the control loop compared to their hysteresis counterparts, although they are more complex to be designed due to the nonlinear nature of the SRM. A PI controller with constant switching frequency has advantages such as easy digital implementation, low ripple current, and simple structure. However, due to the aforementioned nonlinear plant characteristics, good performance and stable operation are difficult to achieve over the full operating range. The main difficulties in both cases are the electrical time constant $(L / R)$ and dynamic disturbance (back EMF) which vary widely with current and rotor position, compromising the performance of the controller [2]. Most of prior papers have been focused on motor design, converter topology, and modulation strategy. Although there are some methods for modeling of SRM drives in literature [5], [9] they do not present rigorous mathematical formulation of the system operation behavior due to the non-linearity in SRM circuit.

In particular, the inner control loop could benefit from additional research. The goal of this paper is to focus on the development of a fully digital implementation of the inner current control loop for high-performance SRM drives operating with high saturation. However, the nonlinear characteristics in the operation of a SRM complicate the analysis and the control of the motor, and limit its applicability to high performance applications such as servo systems.

AC small signal modeling technique utilizing the plant transfer function for the controller design has been widely used in power converters for many years [8]. Without the previous knowledge of the exact transfer function and in fact with time-varying transfer function, it is difficult to design the controller with proper gains and bandwidth. In this paper, a new practical and simple approach for modeling and controlling a SRM current loop and a design methodology for PI current controllers have been developed based on setpoint relay method [10]. By means of the relay control, small oscillations in the output current result. Based on frequency and amplitude measurements of the sustained relay oscillations, two parameters of a first-order-plus-deadtime model at the oscillation frequencies are derived. With the linearized SRM model, the duty cycle for the output transfer function can be derived, and the controller can be designed with sufficient stability margin. The control design is performed without on- 
line iteration and the PI parameters of the current loop are determined analytically and instantaneously using the modified Ziegler-Nichols method [10], and [3], whose advantage lies in the low processing time. Experimental results are included to verify the performance of the proposed method, and an experimental setup was built.

A hardware prototype was implemented with digital signal processor (DSP) to verify the proposed modeling and control approach with the PWM controller switching at $25 \mathrm{kHz}$ to avoid audible noise. The major features of this controller include: 1) constant switching frequency; 2) closed-loop current control; and 3) small current ripple. According to the experimental results, the new simplified mathematical model for the SRM current loop is a suitable tool for control design. The setpoint relay method provides good information about an adequate operation point to determine the parameters of a first-order-plusdeadtime model.

\section{ON-LINE SRM MODELING SCHEME: PROCESS IDENTIFICATION}

\section{A. Describing Function Method}

Describing function analysis is a method that can be used to predict and approximately analyze nonlinear behavior. The basic idea is to approximate a nonlinearity by a linear equivalent and then use different frequency domain techniques to analyze the resulting system [1]. Since a relay is a nonlinearity, this analysis can be adopted. The use of ideal on-off relays limits the identification of the frequency response to only the phase crossover point. This restriction is due to the describing function of the ideal relay being a real valued function. The basic principle of relay feedback method can be explained according to Figure 1. The SRM current transfer function $G_{p}(s)$, which is supposed to be controlled, is regulated firstly using a relay, as seen in Figure 1(a). Thus, an oscillation in the signal with period $T_{u}$ (or angular frequency $\omega_{u}$ ) and amplitude $a$ is generated, as shown in Figure 1(b). The condition for the oscillation to be maintained is the following:

$$
N(a) \cdot G\left(j \omega_{u}\right)=-1
$$

where $N(a)$ is the relay transfer function modeled using the describing function method. Practical implementation of the scheme shown in Figure 1 usually requires the introduction of a hysteresis function in the relay block. This is usually needed in order to avoid multiple zerocrossing points due to the noise in the sensed variable and generalize the method for the estimation of other points in the Nyquist diagrams. When the hysteresis function is used, the describing function of the relay is:

$$
N(a)=\frac{4 d}{\pi a} e^{-j \sin ^{-1}(\varepsilon / a)}
$$

where $d$ is the amplitude of the relay, $\varepsilon$ is the half width of the hysteresis, and $a$ is the amplitude of the resulting oscillations at the output of the plant under the control of the hysteresis relay. The condition stated in (1) is also represented in the Nyquist diagram shown in Figure $1(\mathrm{c})$. Function $1 / N(a)$ can be represented as a straight line parallel to the real axis in the complex plane.
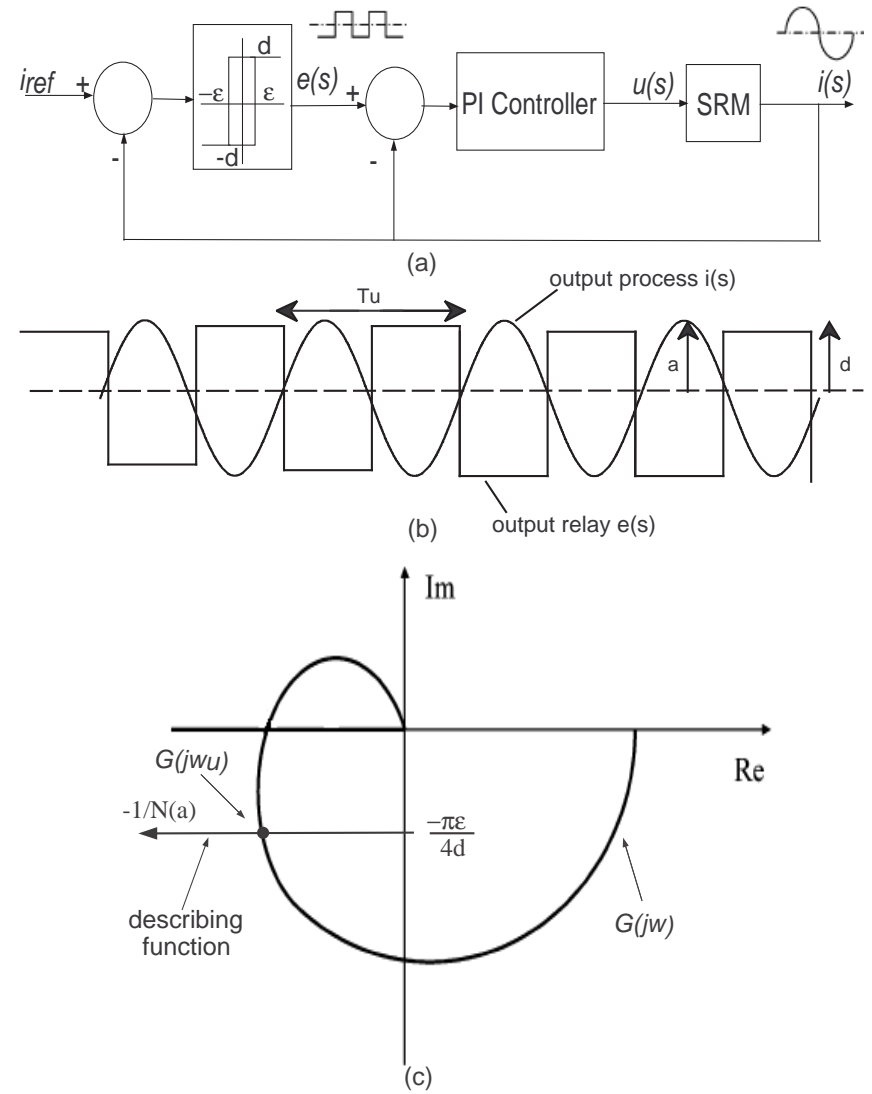

Fig. 1. (a) Block diagram of the process under PI control with setpoint relay. (b) Typical waveforms of relay feedback.

(c) Nyquist diagram of $G_{p}(s)$ and relay block.

In the configuration of Figure 1(a), whose hysteresis relay parameters are $d$ and $\varepsilon$, for the limit cycle conditions with frequency $\omega_{u}$ and amplitude $a$, the operating point approximately satisfies to

$$
\frac{4 d}{\pi a} e^{-j \sin ^{-1}(\varepsilon / a)} G\left(j \omega_{u}\right)=-1
$$

where $G\left(j \omega_{u}\right)$ is the frequency response of the linear process. Hence,

$$
\left|G\left(j \omega_{u}\right)\right|=\frac{\pi a}{4 d}, \angle G\left(j \omega_{u}\right)=-\pi+\sin ^{-1}(\varepsilon / a)
$$

According to (4), for a given set of $d$ and $\varepsilon$, the gain and phase of $G(j \omega)$ can be determined by observing $a$ and $\omega_{u}$ from the resulting limit cycles.

\section{B. Process Model}

In the proposed SRM drive modeling, an asymmetrical bridge converter was used, as shown in Figure 2. In this circuit, each phase winding is represented as one resistance in series with one inductor.

The transfer function from duty cycle to output current can be derived by using ac small signal modeling technique for power converters. When the switches are on, positive voltage $V_{d c}$ is applied to phase winding; when they are turned off, the negative voltage is applied. Assuming the duty cycle as $D$, and considering the average 


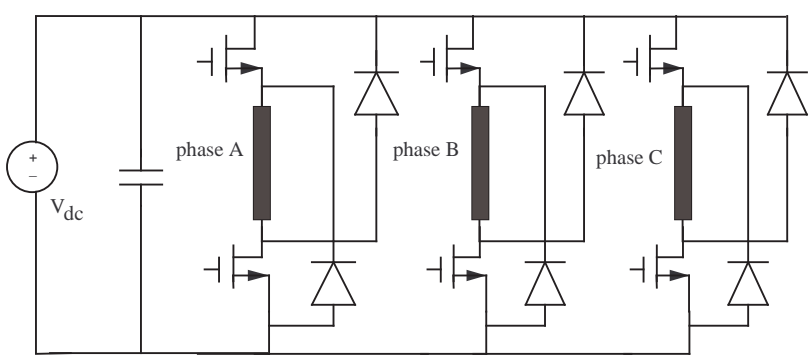

Fig. 2. Three-phase, asymmetrical bridge converter for the SRM.

voltage across the phase winding during one switching period, the transfer function is given by [8]:

$$
G_{i d}(s)=\frac{T_{u o}}{1+s / \omega_{o}}
$$

With

$$
T_{u o}=\frac{2 V_{d c}}{r_{s}+\omega K}
$$

and

$$
\omega_{o}=\frac{r_{s}+\omega K}{L}
$$

where $\omega$ is the speed; $K$ is the gradient of the inductance varying with the rotor position; $L$ is the average inductance over one switching period; $V_{d c}$ is the power supply $\mathrm{dc}$ voltage and $r_{s}$ is the phase resistance. Since parameters $L$ and $K$ vary with the rotor position, the transfer function from duty cycle to output current contains a single moving pole.

The function of the pulse-width modulator is to produce the duty cycle $D$ that is proportional to the output voltage of the compensator. The transfer function of the pulse-width modulator is given by:

$$
\frac{D(s)}{V_{d c}(s)}=\frac{1}{V_{m}}
$$

where $V_{m}$ is the peak voltage of the pulse-width modulator triangular wave.

It can be seen in (5) that the parameters of the transfer function of the converter vary with the rotor position and rotor speed. During the dwell angle of each phase, expressed as $\theta_{d w e l l}$, the inductance $L$ varies between minimum value $L_{u}$ to maximum $L_{a}$, and the inductance gradient $K$ may be either zero or $\frac{L_{a}-L_{u}}{\theta_{d w e l l}}$. A 12/8, $120 V_{d c}$, $2.5 A$, three-phase SRM whose series resistance is $r_{s}=2.4$ ohms, with rotor at speed $220 \mathrm{rpm}$, is employed. The inductance profile for minimum and maximum values are $8 \mathrm{mH}$ and $52 \mathrm{mH}$, respectively, is repeated every 45 mechanical degrees. The open loop transfer function can be given by four expressions as follows, depending on the aforementioned parameters:

$$
\begin{aligned}
G_{i d 1}(s) & =\frac{100}{0.00333 s+1} \\
G_{i d 2}(s) & =\frac{100}{0.02200 s+1} \\
G_{i d 3}(s) & =\frac{72.26}{0.00263 s+1}
\end{aligned}
$$

$$
G_{i d 4}(s)=\frac{72.26}{0.01700 s+1}
$$

Figure 3 shows some regions in the Nyquist diagram for two of the aforementioned transfer functions, which are used as a base for identification and control. Such functions are limited because they depend on the previous knowledge of the motor parameters. However, this limitation can be overcome by identification methods.

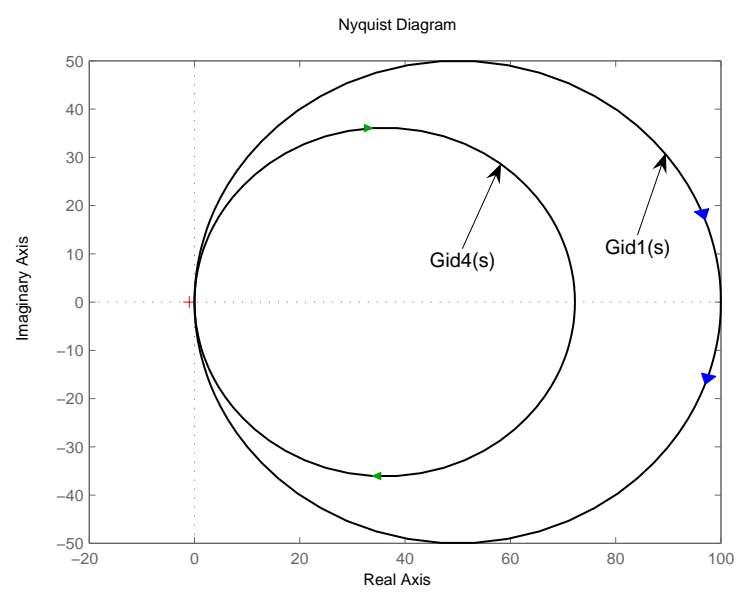

Fig. 3. Nyquist diagram for the transfer function models.

\section{SRM Drive Identification by Setpoint Relay under PI Control}

The phase current transfer function can be modeled on-line using the setpoint relay experiments. This online modeling technique does not require a priori knowledge of SRMs electromagnetic characteristics. The control scheme comprehends a relay and an extra feedback signal to the setpoint, as shown in Figure 1. The basic idea of the setpoint relay is similar to that of relay feedback experiment [3] except that it has the advantage of oscillating within a safe "operation zone" due the performance of the PI controller in forward loop and the existing control system is always in closed-loop during the tuning. The control parameters are determined from the knowledge of the critical gain and the critical period.

Assuming that the gain of the relay is $K_{r}$, which corresponds to its describing function, the transfer function of the controller is $G_{c}(s)$ and the transfer function of process is $G_{p}(s)$, the error $e(s)$ can be calculated as:

$$
e(s)=K_{r} i_{r e f}-\left(1+K_{r}\right) v(s) .
$$

where $i_{r e f}$ is the setpoint and $v(s)$ is the control variable. Hence the block diagram can be rearranged as shown in Figure 4. The loop transfer function is $(1+$ $\left.K_{r}\right) G_{p}(s) G_{c}(s)$. The equivalent gain or describing function for the relay element is [7]:

$$
K_{r}=\frac{4 d}{\pi \sqrt{a^{2}-\varepsilon^{2}}}
$$

Although only one Nyquist curve point can be identified by relay without hysteresis, two unknown parameters 


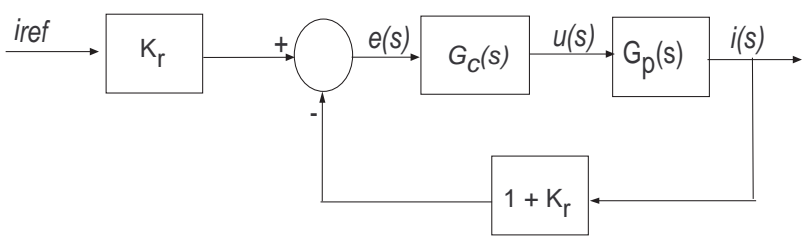

Fig. 4. Block diagram of the setpoint relay.

in the process model could be determined from gain and phase conditions, according to Figure 1(c). Therefore, the transfer function from the duty cycle to the output voltage represented by (5) plus deadtime model given by [3] is adopted as follows:

$$
G_{p}(s)=\frac{K_{p} e^{-\theta s}}{\tau s+1}
$$

where $K_{p}$ is the process gain, $\tau$ is the time constant, and $\theta$ is the dead time.

It is important to mention that (5) corresponds to an exact expression for the SRM converter proposed in [8], and it was also used to interpret the relay autotuning method by [3]. This transfer function will be applied to the SRM drive shown in Figure 1. Let us consider a PI controller with the following transfer function:

$$
G_{c}(s)=K_{c}\left(1+\frac{1}{T_{i} s}\right) .
$$

where $T_{i}$ is the integral time. Then, the system is forced to oscillate at the resonant frequency, $\omega_{u}$. When sustained oscillation is developed, it gives:

$$
\arg \left[\left(1+K_{r}\right) G_{p}\left(j w_{u}\right) G_{c}\left(j w_{u}\right)\right]=-\pi .
$$

and

$$
\left|\left(1+K_{r}\right) G_{p}\left(j w_{u}\right) G_{c}\left(j w_{u}\right)\right|=1 .
$$

where $w_{u}$, is the oscillation frequency. From (15) one can obtain:

$$
\tau=\frac{\left.\left(1+K_{r}\right) K_{p} \sqrt{T_{i}^{2} \omega_{u}^{2}+1}\right) .}{T_{i} \omega_{u}^{2}} .
$$

Analogously, the deadtime estimate in the model given by (12) can be obtained from (14) as:

$$
\theta=\frac{\left[\frac{\pi}{2}+\tan ^{-1}\left(T_{i} \omega_{u}\right)-\tan ^{-1}\left(T \omega_{u}\right)\right]}{\omega_{u}} .
$$

Defining

$$
K_{u}=\frac{2 \pi \tau}{T_{u}}
$$

and

$$
T_{u}=\frac{2 \pi}{\omega_{u}}
$$

One can easily notice from the above assumptions that $K_{u}$ is the gain that brings the system to stability boundary. The ultimate gain $K_{u}$ and the ultimate period $T_{u}$ are easily found from a relay experiment [7].

The developed modeling is used in the design of the controllers. Figure 5 shows the block diagram of the model used for relay setpoint simulation.

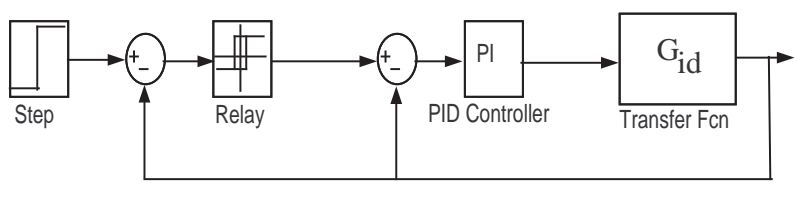

Fig. 5. Diagram block of relay setpoint simulation.

For relay setpoint simulation, the SRM transfer functions given by (7) and (10) were employed. The first approximation of transfer function with parameters $\varepsilon=0.2$ and $d=1.5$ was adopted as the relay setpoint when sustained oscillation is developed. Figure 6 shows the relay setpoint simulation results with PI parameters given by $K_{c}=100, T_{i}=2.22 \times 10^{-4} \mathrm{~s}$, and step current reference of $2.5 \mathrm{~A}$.
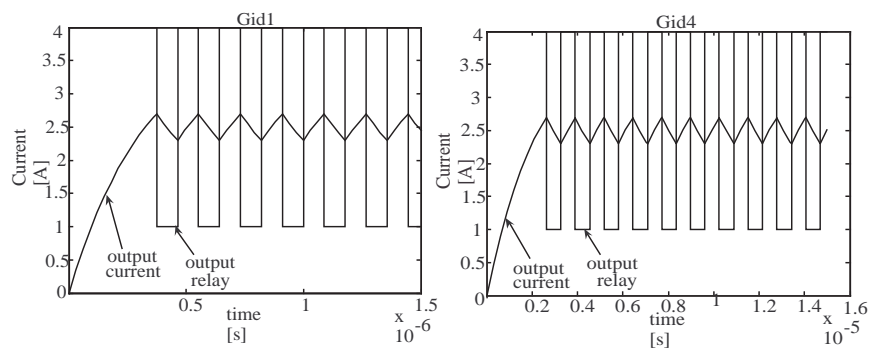

Fig. 6. Relay setpoint simulation for the transfer functions $G_{i d 1}$ and $G_{i d 4}$.

\section{CURRENT CONTROLLER DESIGN}

To verify the proposed method for the modeling of the SRM current loop, then based on the estimates of deadtime and time constant, it is possible to use the ZieglerNichols reaction curve formula to calculate the PI parameters directly, as generally this results in poor tuning of the PI parameters. In this paper, the modified ZieglerNichols method proposed in [7] was adopted. Initially, one must choose an arbitrary point on the Nyquist curve of the open loop system as:

$$
G_{p}\left(j \omega_{o}\right)=r_{a} e^{j\left(\pi+\phi_{a}\right)}
$$

where $r_{a}$ and $\phi_{a}$ correspond to magnitude and phase of the arbitrary point.

The controller parameters can be designed to provide specific phase and gain in terms of the transfer functions:

$$
G_{l}\left(j \omega_{o}\right)=r_{b} e^{j\left(\pi+\phi_{b}\right)}
$$

where $r_{b}$ and $\phi_{b}$ correspond to magnitude and phase of the design, respectively.

The frequency response of the controller is:

$$
G_{c}\left(j \omega_{o}\right)=r_{c} e^{j \phi_{c}}
$$

where $r_{c}$ and $\phi_{c}$ correspond to magnitude and phase of the controller, respectively.

The loop transfer function for the compensated system can be calculated from:

$$
r_{b} e^{j\left(\pi+\phi_{b}\right)}=r_{c} e^{j \phi_{c}} r_{a} e^{j\left(\pi+\phi_{a}\right)}
$$


Assuming $r_{a}=1 / K_{u}$ at the crossover frequency, the PI controller parameters are given by:

$$
\begin{aligned}
K_{c} & =K_{u} r_{b} \cos \left(\phi_{b}\right) . \\
T_{i} & =-\frac{T_{u}}{2 \pi \tan \left(\phi_{b}\right)} .
\end{aligned}
$$

where $r_{b}$ and $\phi_{b}$ are the specified argument and phase, respectively, i.e. the design parameters.

Figure 7 shows the closed loop response when a PI controller is tuned by modified Ziegler-Nichols. According to [7], PI tuning has been performed with the desired loop transfer function represented by:

$$
G_{l}\left(j 7.865 \times 10^{3}\right)=0.41 e^{j(\pi+60)}
$$

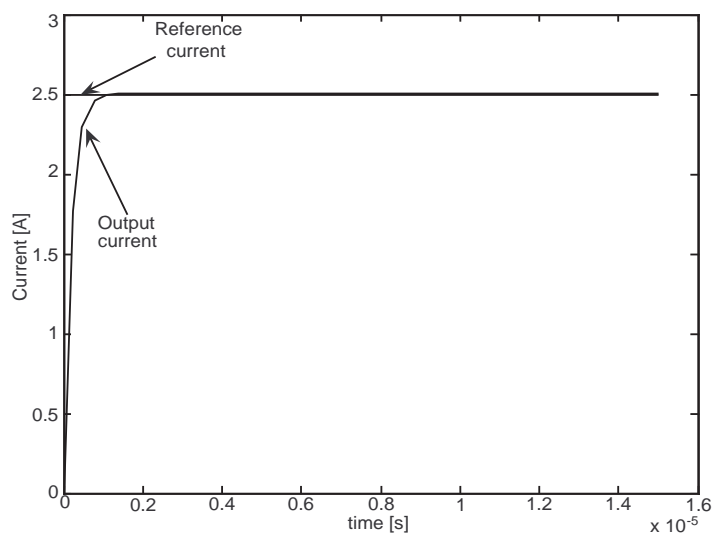

Fig. 7. PI controller based on modified Ziegler-Nichols.

\section{EXPERIMENTAL RESULTS}

In order to verify the proposed method to model the SRM current loop, a hardware prototype was implemented with digital signal processor TMS320F2812 of Texas Instruments as the switching frequency is $25 \mathrm{kHz}$, considering the block diagram in Figure 8.

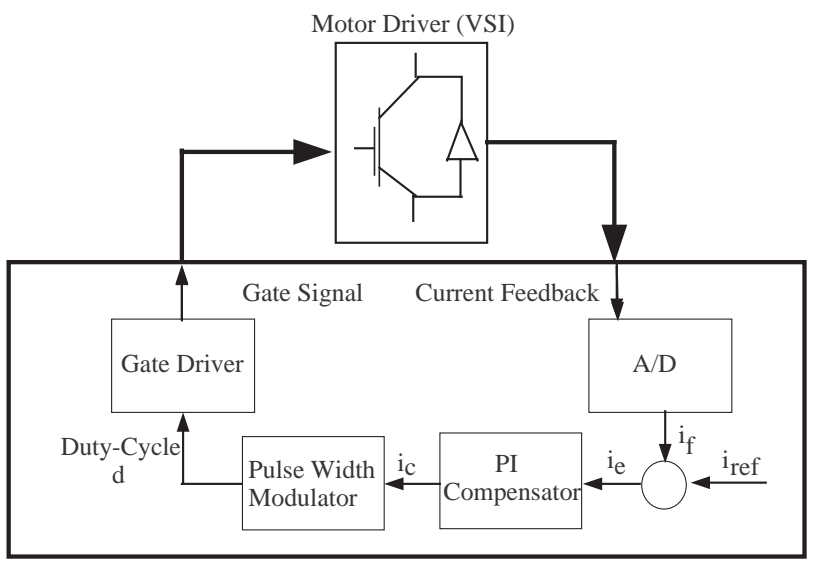

DSP Current Controller

Fig. 8. DSP controller-based SRM drive system configuration.
In this test bench setup, the SRM presented in section $I I . B$ was assumed in relay setpoint identification. The inductance profile with a minimum and a maximum is repeated every 45 mechanical degrees. Because only one phase is allowed to conduct at any time, there is no overlap between the firing of two phases. Parameter $\theta_{\text {dweel }}$ is 15 mechanical degrees for each phase. All three-phase currents were sensed and fed through 16-bit analog-todigital (A/D) converters. The complete controller including PI compensator and PWM control was implemented in DSP coding. The experimental setup shown in Figure 9 was developed, as the current and voltage sampling rate is $40 \mu s(25 \mathrm{kHz})$.

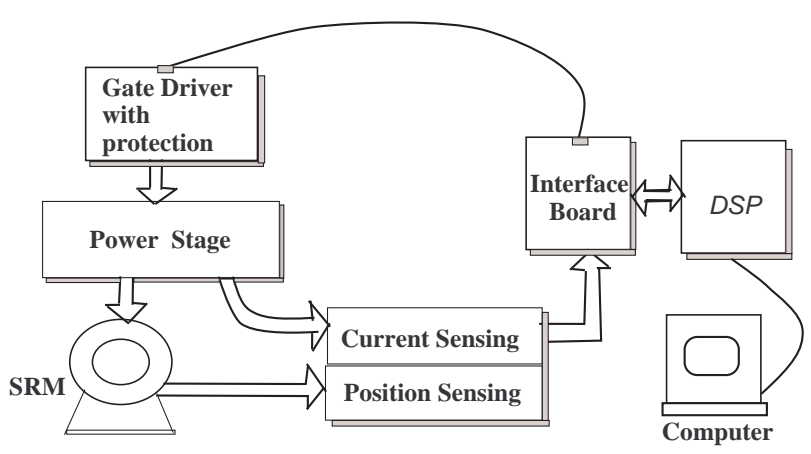

Fig. 9. Experimental system.

In order to observe the open loop behavior of the system under the same voltage, current, and speed conditions, experimental results regarding the dc link current and the three-phase currents were obtained, as shown in Figure 10. It can be seen that the dc link current corresponds to the sum of the three-phase currents. Figure 10 also shows that the dc link current presents some dip during the phase transitions state, which is due to different slopes in the incoming and outgoing phase currents. The waveform illustrates the torque ripple problem in threephase motors, considering that torque is proportional to the current and is often controlled employing an inner current loop. Therefore Figure 10 gives an insight of the torque problem in SRM system, i.e., the current loop is supposed to be efficiently controlled for good torque characteristic.

During the identification phase, first the setpoint relay experiments for SRM drives with the following values were carried out, where phase current through windings is $2.5 A$, hysteresis amplitude is $d=1.5$, and hysteresis width is $\varepsilon=0.2$. Considering the same speed and dc link voltage conditions of the previous test, the sustained oscillation for the SRM phases is shown in Figure 11. From the obtained results, the output variable $i$ (corresponding to the winding currents), oscillation amplitude $a$, and period $T_{u}$ were measured. Then the parameters in (12) were obtained based on expressions (16) and (17). From Figure 11 it was found $\tau=0.0861 \mathrm{~s} ; \theta=0.0195 \mathrm{~ms}$; $T_{u}=0.000799 \mathrm{rad} ; K_{u}=859.2$, and amplitude $a=0.35$. The parameters of the PI controller in Figure 1, were established as $K_{c}=100$ and $T_{i}=2.22 \times 10^{-4} \mathrm{~s}$. The gain was also determined in steady state. As a result of this identification procedure, the estimated tranfer function 


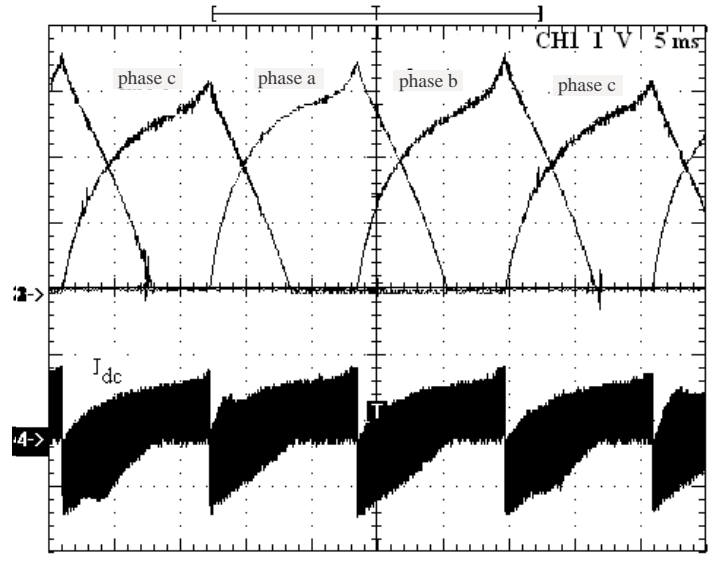

Fig. 10. DC link current and the three-phase currents under unstable current-loop condition.

for SRM loop current given by (11) is:

$$
G_{p}(s)=\frac{89 e^{-0.2 m s}}{0.1092 s+1} .
$$

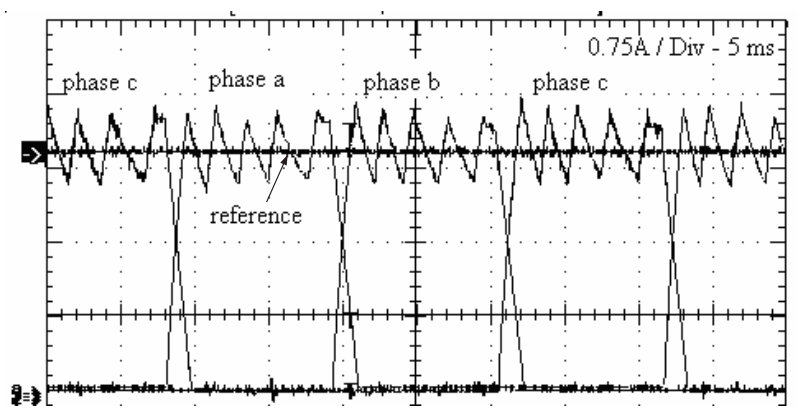

Fig. 11. Output of relay setpoint: phases A,B, and C.

Figure 12 shows the Nyquist diagram of $G_{p}(s)$ and the transfer functions given by physical parameters present in (7) and (10).

Since the transfer function varies with the rotor position, it is impossible to design a fixed PI compensator to maintain constant gain and phase margin at different rotor position. There are two ways to design a compensator to make system stable. One is to design a fixed compensator at worst case to make system stable for all rotor position. Designing a time varying compensator with rotor position to maintain stable performance along the time employing adaptative control is another alternative.

The relay method identifies two points of SRM current loop transfer function. With this approach it is possible to identify a transfer function within the interval (Gid1 to Gid4, seen in Figure 12) that provides better process dynamic representation. Then the time constant $\tau$ considers the effect the parameters variation with rotor position and rotor speed. For the speed change in relay setpoint method, other operating points exist, mainly the reference current.

One can see from Figure 12 that the relay setpoint method provides appropriate results using the ac small

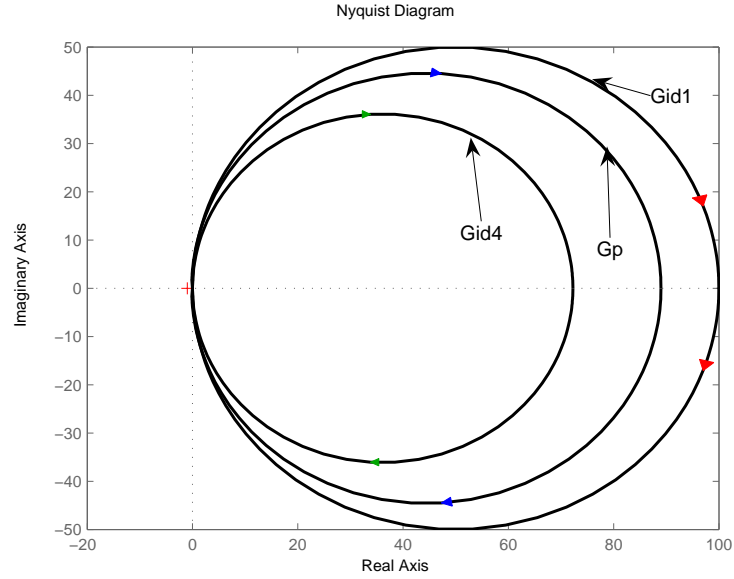

Fig. 12. Nyquist diagram of identified function by two method.

modeling technique described by (5). This transfer function by relay setpoint method is used as a base for the design of controllers that will be implemented experimentally to validate the proposed method. Therefore the controllers are supposed to operate around the operating point defined during the identification process. This point is defined from the current and speed parameters used during the experiment, that are $i_{r e f}=2.5 \mathrm{~A}$ and $\omega_{r}=220 \mathrm{rpm}$.

To validate this approach further, extensive experimentation was performed and far more relevant results were obtained. Then other tests with different values for $\varepsilon$ with hysteresis amplitude fixed at $d=1.5$ using the same existing PI controller setting were obtained. Some cases are shown in Table I.

TABLE I

Identification Results from Setpoint Relay

\begin{tabular}{ccccc}
\hline$\varepsilon$ & $T_{u}[\mathrm{rad}]$ & $K_{u}$ & $\tau[\mathrm{ms}]$ & $\theta[\mathrm{ms}]$ \\
\hline 0.05 & 0.0007 & 413.38 & 0.0460 & 0.176 \\
\hline 0.10 & 0.0074 & 552.89 & 0.0655 & 0.187 \\
\hline 0.20 & 0.0080 & 859.20 & 0.1092 & 0.200 \\
\hline
\end{tabular}

In equation (26), since the current loop time delay $(\theta)$ is smaller than the time constant, the time delay has been disregarded for modeling purposes. Assuming a $\mathrm{ZOH}$ and a sampling rate of $40 \mu \mathrm{s}$, the discrete equivalent of equation $(26)$ is:

$$
G_{p}(z)=\frac{0.003649 z^{-1}}{1-0.9964 z^{-1}}
$$

This equation (27) was used to implement the SRM current controller. Thus, considering $\varepsilon=0.2$, and in order to verify the effectiveness of proposed current-loop controller design methodology, the estimated parameters are used for auto-tuning of the PI controller by modified ZieglerNichols method employing $r_{b}=0.29$ and $\phi_{b}=46^{\circ}$, with the obtained gain proportional $K_{c}=173.073$, integral time $T_{i}=0.123 \mathrm{~ms}$, and operating frequency $\omega_{u}=1.2517 \times 10^{3} \mathrm{rad} / \mathrm{s}$. According to Figure 13, a 
basic implementation is supposed to take into account a load in the SRM shaft (represented by a dc motor).

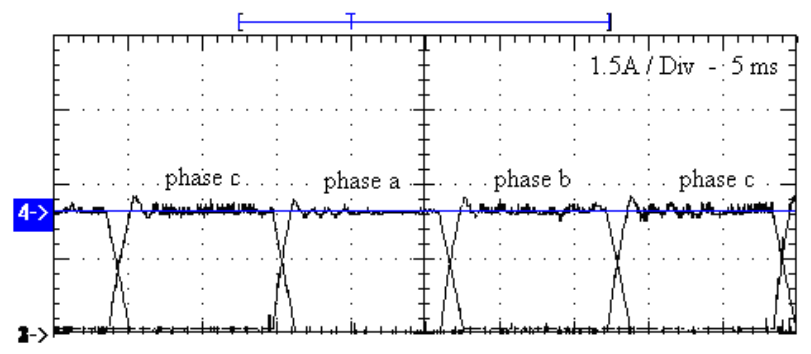

Fig. 13. Currents through SRM phases using PI controller.

In order to demonstrate the usefulness of the proposed method, experimental tests were repeated for different setpoints varying both current (from $2.5 A$ to $3.5 A$ ) and rotor speed (220 rpm and $300 \mathrm{rpm}$ ), respectively. Figures 14 and 15 show the current waveforms with the PI controller.

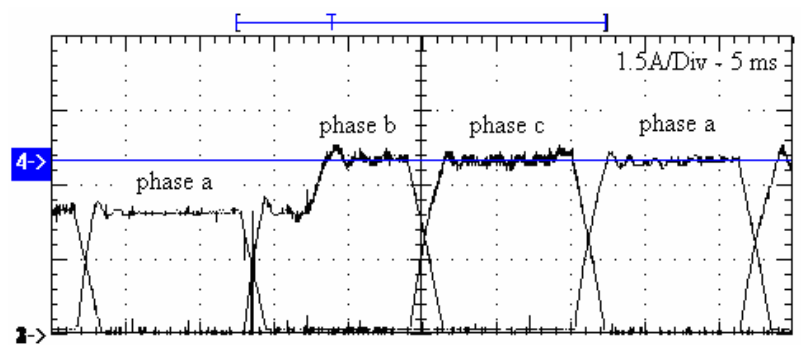

Fig. 14. Currents through SRM phases using PI controller and setpoint change $(\omega=220 \mathrm{rpm})$.

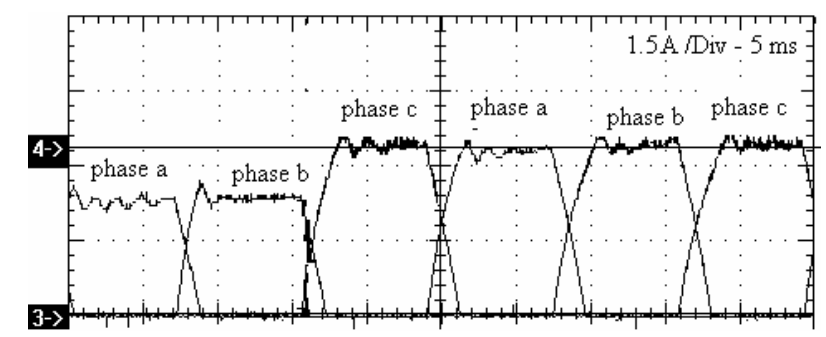

Fig. 15. Current waveforms through SRM phases using PI controller and setpoint change $(\omega=300 \mathrm{rpm})$.

Figure 16 shows multiple cycles of the dc link ripple current component and the three-phase currents with PI controller, which are 120 degrees apart.

By using an iterative procedure, an adaptive control that presumes an identification method can be considered. The tuning of PI parameters is obtained directly by including the controller in the relay feedback and by adjusting the controller parameters based on the specified performance.

The application of setpoint relay was performed at relative low speed for the SRM mainly due to two reasons: the first one is because the current control is an issue only observed at low speed, where current normally is limited

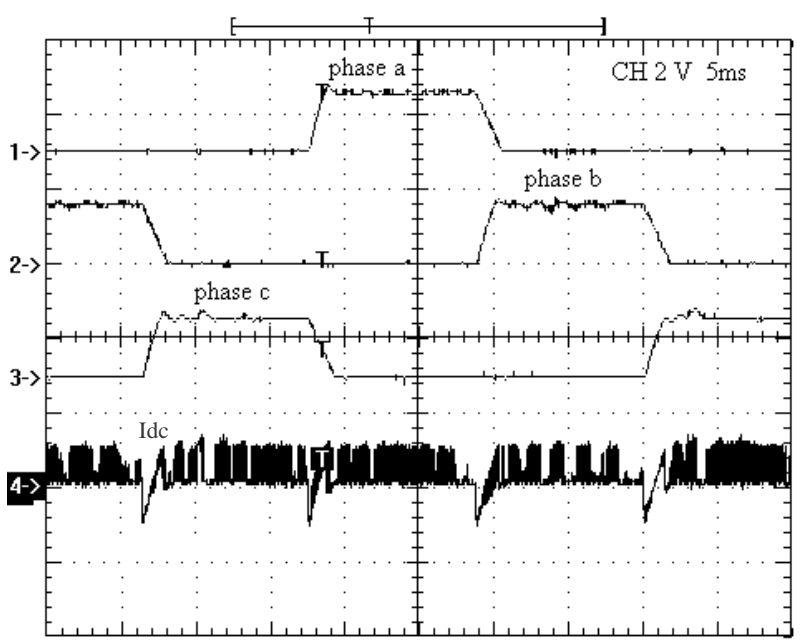

Fig. 16. DC link current and the three-phase currents.

by chopping. The use of single pulse control is typically used at speeds equal or greater than the nominal specification. The second one is because sustained oscillation can not be developed due to the speed increase, as the relay setpoint method may not be suitable.

\section{CONCLUSION}

The main contribution of this paper is the use of relay feedback for identifying the SRM drive current loop transfer function. Moreover, a simple autotuning technique for SRM drives using the PWM controller was also introduced. The identification is accomplished in the frequency domain and the results are used in the design of a PI control loop based on gain and phase specifications. The method is an extension of the relay feedback control where relevant information are extracted from limit cycles established by relay setpoint method and the describing function method is used for modeling purposes. The method has the advantage of keeping the machine operation within a "safe operation zone" since existing control system is always in closed-loop during the identification phase. The proposed SRM drive modeling scheme does not require the previous knowledge of motor characteristics. To verify the proposed method, the modified Ziegler-Nichols PI tuning was used to calculate the controller parameters. Experimental results have been presented and demonstrate the feasibility of the SRM drive modeling and control scheme.

\section{REFERENCES}

[1] H. K. Khalil, "Nonlinear Systems", Prentice Hall, London, 1990.

[2] G. Schroder and J. Bekiesch, "Current Control for the Switched Reluctance Motor with Enhanced Performance", Proc. Eur. Conf. IEEE Power Electronics and Applications, september, 2005, pp. 1-8.

[3] I. Nascu and R. De Keyser, "A Novel Application of Relay Feedback for PID Auto-tuning", Proc. of the American Control Conference, CSD03-Conf. on Control systems Design, Bratislava, Slovak Republic, 2003. 
[4] L. L. N. dos Reis and R. N. C. Almeida and A. A. R. Coelho and O. M. Almeida and J. C. T. Campos, "Current Control of Switched Reluctance Motor Based on Generalized Minimum Variance Controller", Proc. of the 2007 American Control Conference (ACC), New York, july, pp. 3541-3545, 2007.

[5] I. Husain and S. A. Hossain, "Modeling, Simulation, and Control of Switched Reluctance Motor Drives", IEEE Transactions on Industrial Electronics, vol. 52, no. 6, May 2005, pp. 1625-1634.

[6] Z. Lin and D.S. Reay and B.W. Williams and X. He, "High Performance Current Control for Switched Reluctance Motors with On-line Modeling", Proc. IEEE Power. Elec. Spec. Conf. Annual Meeting PESC, Aachen, 2004.

[7] K. J. Åström and T. Hägglund, "Advanced PID Control", ISA-Instrumentation, Systems, and Automation Society, USA, 2006.

[8] G. Zhu and H. Wei and P. Kornetzky and I. Batarseh, "Small-Signal Modeling of a Single-Switch AC/DC Power-Factor-Correction Circuit", IEEE Trans. on Power Eelctronics, vol. 14, no. 6, 1999, pp. 1142-1148.

[9] X. D. Xue and K. W. E. Cheng and S. L. Ho and D. Sutanto, "Precise Analytical Modelling Magnetic Characteristics of Switched Reluctance Motor Drives Using Two-Dimensional Least Squares", IEEE Power Electronics Specialist Conference, PESC03 34th Annual, 2003, pp. 416-421.

[10] R. Luo and S. Joe Qin and D. Chen, "A New Approach to Closed-Loop Autotuning for PID Controllers", Proceedings of the American Control Conference, June 1998, pp. 348-352.

\section{BIOGRAPHIES}

Laurinda L. Nogueira dos Reis Received the B.Sc. degree in Electrical Engineering from the Federal University of Ceara, Fortaleza, Brazil in 1979, and the M.Sc. degree in Electrical Engineering from the Federal University of Paraíba, Campina Grande, Brazil in 1984, respectively. She is currently pursuing the Ph.D. degree at the Federal University of Santa Catarina, Florianópolis, Brazil. She has been a professor with the Federal University of Ceara since 1979. Her research interest areas are electrical machine drives, advanced control techniques, and nonlinear systems.

Antonio Augusto Rodrigues Coelho Received the B.Sc. degree in Electrical Engineering from the Federal University of Pará, Belém, Brazil in 1979, the M.Sc. degree in Electrical Engineering from the Federal University of Santa Catarina, Florianópolis, Brazil in 1983, and the Ph.D. degree in Electrical Engineering from the State University of Campinas, Campinas, Brazil in 1991. He currently is an Associate Professor. His research interest areas are systems identification, predictive and adaptive control, computational intelligence in control, multivariable control, applied digital PID control, and control of mechatronic systems.

Otacílio da Mota Almeida Received the B.Sc. degree in Electrical Engineering from the Federal University of Ceará, Fortaleza, Brazil in 1985, the M.Sc. degree in Electrical Engineering from the State University of Campinas, Campinas, Brazil in 1998, and the Ph.D. degree in Electrical Engineering from the Federal University of Santa Catarina, Florianópolis,
Brazil in 2005. He has been a professor with the Federal University of Ceara since 1991. His research areas are electric machinery, electrical machine drives, advanced control techniques for electromechanical systems, and nonlinear systems.

Rômulo Nunes Almeida Was born in Fortaleza, Brazil, in 1982. Electrical technician by Centro Federal de Educação Tecnológica do Ceará, Fortaleza, Brazil in 2001. Received the B.Sc. degree and the M.Sc degree in Electrical Engineering from the Federal University of Ceará, Fortaleza, Brazil in 2005 and 2007 respectively. He has been a researcher in Grupo de Pesquisa em Automação e Robótica at Federal University of Ceará, fortaleza, Brazil since 2005. His research interest áreas are electric machinery, electrical machine drives, embedded systems, advanced control techniques and nonlinear systems. 